\title{
A Bridge Circuit for Temperature Drift Cancellation
}

Masaru Matsuno, Shinobu Adachi, Masakatsu Nakayama, and Kenzo Watanabe

\begin{abstract}
A bridge circuit is developed for cancelling temperature drifts of resistive sensors. It consists basically of two haif bridges with a common reference arm, each one followed by a difierential amplifier. The gains of the amplifiers are adjusted such that the half-bridge output voltages due to temperature variation of sensors cancel each other. This scheme allows a compensation for a large mismatch in the temperature sensitivity of sensors without degrading their inherent sensitivities. Experimental results are also presented to demonstrate the validity of this concept.
\end{abstract}

\section{INTRODUCTION}

Since its invention by Wheatstone, the resistance bridge has a history longer than 130 years. This old bridge, originally used for high-precision resistance measurements, is still actively used [1], [2]; it is now applied extensively to sensing such physical and chemical quantities as temperature, humidity, gas concentration, strain, pressure, and position. Typical elements incorporated into these sensing bridges are platinum wires, thermistors, piezo-, and magneto-resistors. The resistance of these sensors changes not only よって書かれた論文のため、未掲載 with these measurands but also with temperature. Therefore, except for temperature measurements, reducing the temperature drift is of the utmost concern in designing a high-sensitivity sensing bridge [3], [4].

Resistive sensors are usually arranged in a quarter, half, or full bridge [3]. In a quarter bridge, a passive sensor which is made sensitive only to temperature is connected in series with the sensor to cancel the effect of temperature on the active sensor. In a half or full bridge, the balance against temperature is performed by using sensors with matched temperature coefficients. These conventional methods for temperature compensation postulate that the output voltage between the bridge nodes must be processed by one differential or instrumentation amplifier. If the output voltages at the nodes are amplified separately, then the apparent temperature sensitivity of each bridge arm can be adjusted by the gain of each amplifier. This adjustment is independent of the bridge balance and thus will not degrade its inherent sensitivity. Based on this idea, a new architecture is developed.

\section{Bridge Configuration}

Fig. 1 shows a block diagram of the bridge circuit for cancelling temperature drifts. Here, $S_{1}$ to $S_{4}$ form a conventional sensing bridge, and $R_{\mathrm{A}}$ and $R_{\mathrm{B}}$ form a voltage divider to produce a reference voltage $E_{2}$. Therefore, this scheme can be regarded as consisting of two half bridges each followed by a differential amplifier. The imbalance output of each bridge at temperature $T$ can be expressed as

$$
\begin{aligned}
& E_{1}-E_{2}=\zeta_{12} x+\alpha_{12}\left(T-T_{o}\right) \\
& E_{3}-E_{2}=\zeta_{32} x+\alpha_{32}\left(T-T_{o}\right)
\end{aligned}
$$

Manuscript received September 29, 1992; revised February 2, 1993.

M. Matsuno and S. Adachi are with the Gas Instrument Development Center, Yazaki Meter Corp., Futamata-cho, Tenryu, 431-33 Japan.

M. Nakayama and K. Watanabe are with the Research Institute of Electronics, Shizuoka University, Hamamatsu, 432 Japan.

IEEE Log Number 9209225. 


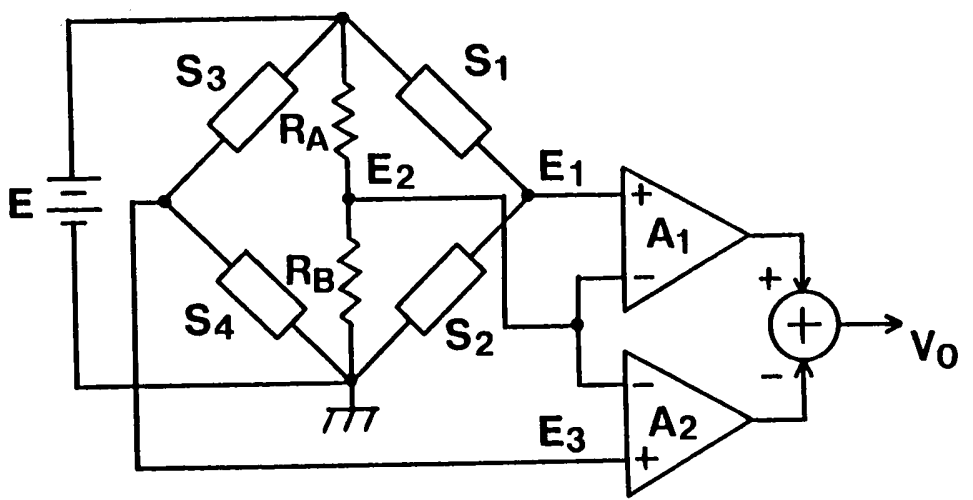

Fig. 1. A bridge configuration for temperature drift cancellation.

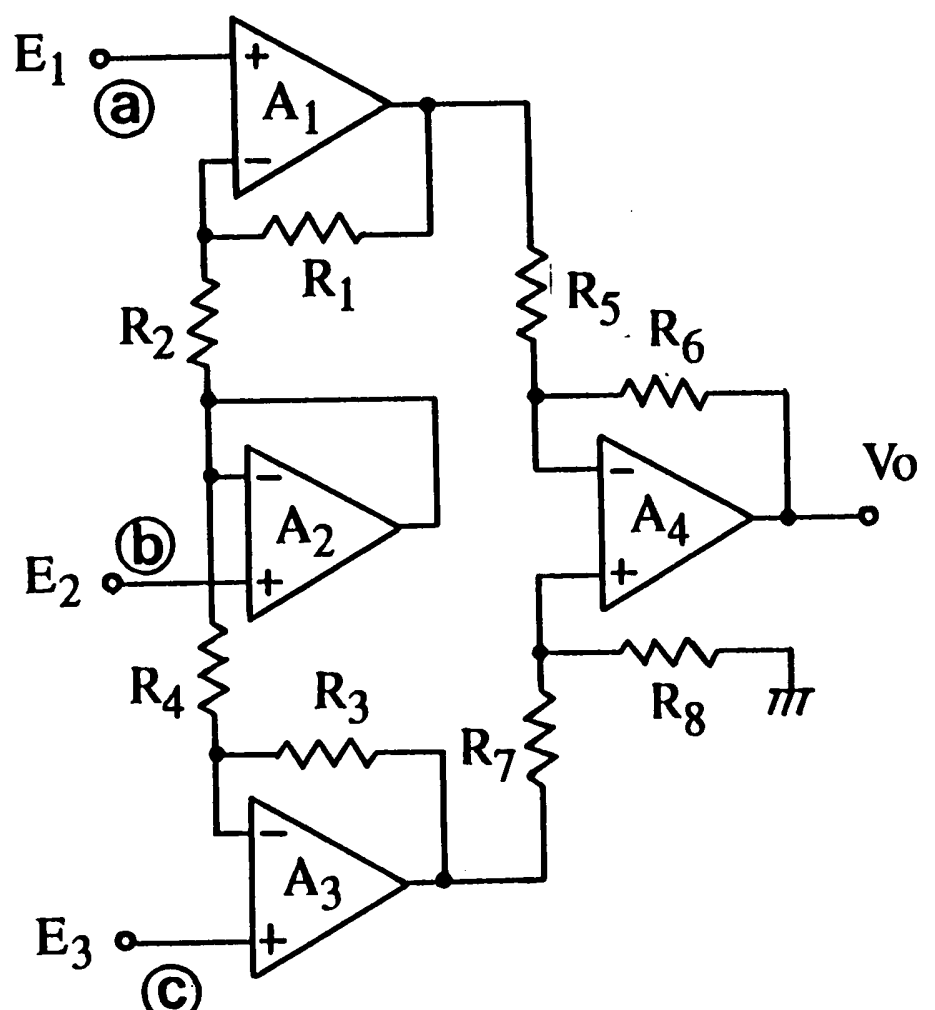

Fig. 2. A circuit diagram of the differential amplifiers.

where $\zeta$ is the inherent sensitivity of the bridge to a measurand $x$, $\alpha$ is the temperature sensitivity, and $T_{o}$ is the temperature at which the bridge is balanced. The output voltage $V_{o}$ of the subtractor is then given by

$$
V_{o}=\left(G_{1} \zeta_{12}-G_{2} \zeta_{32}\right) x+\left(G_{1} \alpha_{12}-G_{2} \alpha_{32}\right) \Delta T
$$

where $G_{1}$ and $G_{2}$ are differential gains of $A_{1}$ and $A_{2}$, respectively, and $\Delta T=T-T_{o}$. It follows, therefore, that if

$$
G_{1} / G_{2}=\alpha_{32} / \alpha_{12}
$$

then no temperature drifts appear at the output.

The temperature balance condition (4) assumes temperature sensitivities of the same polarity. If different, the outputs of the two differential amplifiers should be added to get the same condition.

A practical circuit of differential amplifiers is shown in Fig. 2. Assuming that all the op-amps are ideal and that the differential stage formed by $A_{4}$ is balanced, i.e.,

$$
R_{5} R_{8}=R_{6} R_{7}
$$

one can easily derive the expression for the output as

$$
\begin{aligned}
V_{o}= & \frac{R_{6}}{R_{5}}\left[\left\{\left(1+\frac{R_{3}}{R_{4}}\right) \zeta_{32}-\left(1+\frac{R_{1}}{R_{2}}\right) \zeta_{12}\right\} x\right. \\
& \left.+\left\{\left(1+\frac{R_{3}}{R_{4}}\right) \alpha_{32}-\left(1+\frac{R_{1}}{R_{2}}\right) \alpha_{12}\right\} \Delta T\right] .
\end{aligned}
$$

The temperature balance condition is thus given by

$$
\frac{1+\frac{R_{3}}{R_{4}}}{1+\frac{R_{1}}{R_{2}}}=\frac{\alpha_{12}}{\alpha_{32}}
$$

\section{Temperature Drift Rejection Ratio}

The temperature balance condition (7) can be established independently of the bridge balance, but is affected by the offset voltages of op-amps. Their effect will be next analyzed with reference to the balance procedure.

With sensors unstimulated, the bridge circuit is balanced by the following steps.

1) Apply a dc voltage much larger than the offset voltages of op-amps to the input terminals $a, b$, and c shown in Fig. 2, trim $R_{8}$ such that the output $V_{o}=0$. This establishes the balance condition (5).

2) Keeping sensors at some temperature $T_{o}$ and applying the bridge output $E_{3}$ to the terminal c and $E_{2}$ to both terminals a and $\mathrm{b}$, adjust $E_{2}$ by means of a potentiometer, which is connected between $R_{A}$ and $R_{B}$, such that the output $V_{o}=0$. Then, the offset voltages $V_{O S_{1}}, V_{O S_{2}}$, and $V_{O S_{3}}$ of op-amp $A_{1}, A_{2}$, and $A_{3}$, respectively, are balanced or compensated for by the imbalance voltage $\Delta_{32}$ between $E_{3}$ and $E_{2}$, i.e.,

$$
\left(1+\frac{R_{3}}{R_{4}}\right)\left(\Delta_{32}+\epsilon_{32}\right)-\left(1+\frac{R_{1}}{R_{2}}\right) \epsilon_{12}=0
$$

where

$$
\begin{aligned}
& \epsilon_{32}=V_{O S_{3}}-V_{O S_{2}} \\
& \epsilon_{12}=V_{O S_{1}}-V_{O S_{2}} .
\end{aligned}
$$

3) Applying the bridge outputs $E_{1}, E_{2}$, and $E_{3}$ properly to the terminals a, b, and c, adjust $E_{1}$ by means of a potentiometer connected between $S_{1}$ and $S_{2}$ such that $V_{o}=0$. This establishes $E_{1}=E_{2}$, for the offset voltages of op-amps have already been balanced by the imbalance voltage $\Delta_{32}$ in step 2 ).

4) Keeping sensors at some other temperature $T_{m}$, adjust the gain, say $G_{1}$, by trimming $R_{1}$ such that $V_{o}=0$. Then the following relation is obtained

$$
\begin{aligned}
\left(1+\frac{R_{3}}{R_{4}}\right)\left(\alpha_{32} \Delta T_{m}+\Delta_{32}+\epsilon_{32}\right) & \\
& -\left(1+\frac{R_{1}+\delta R_{1}}{R_{2}}\right)\left(\alpha_{12} \Delta T_{m}+\epsilon_{12}\right)=0
\end{aligned}
$$

where $\delta R_{1}$ is the trimmed resistance of $R_{1}$ and $\Delta T_{m}=T_{m}-$ $T_{o}$.

It is clear from (11) that, as they enter in the temperature balance condition, the offset voltages of op-amps cause an imperfect cancellation of the temperature drift. The residual drift $V_{o}(T)$ at tem- 


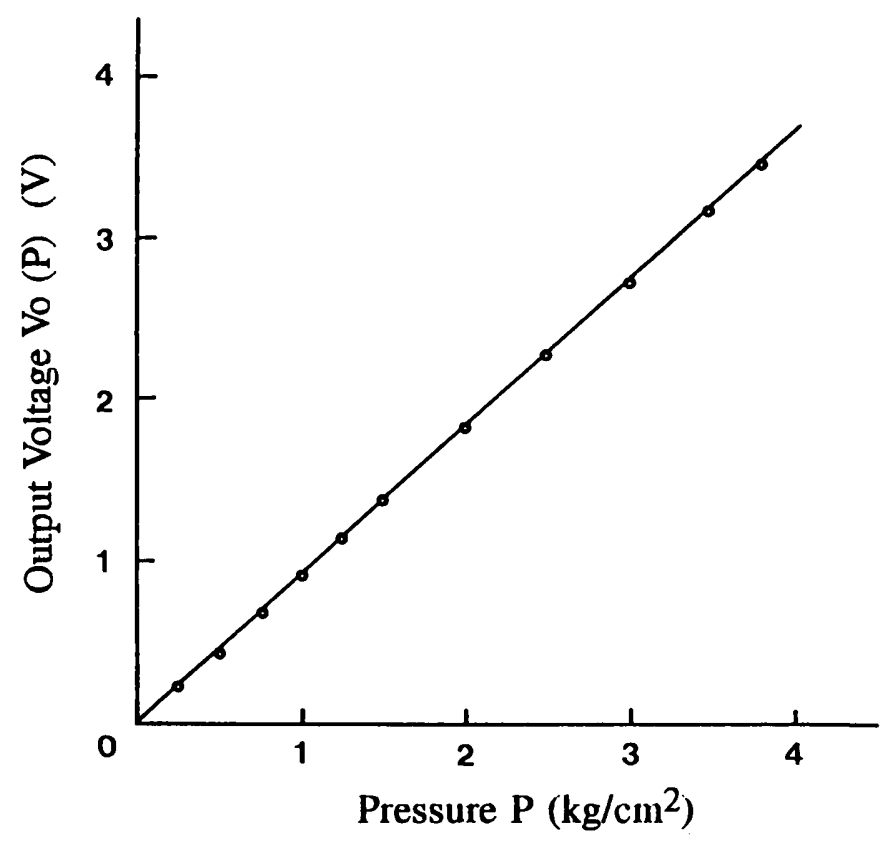

(a)

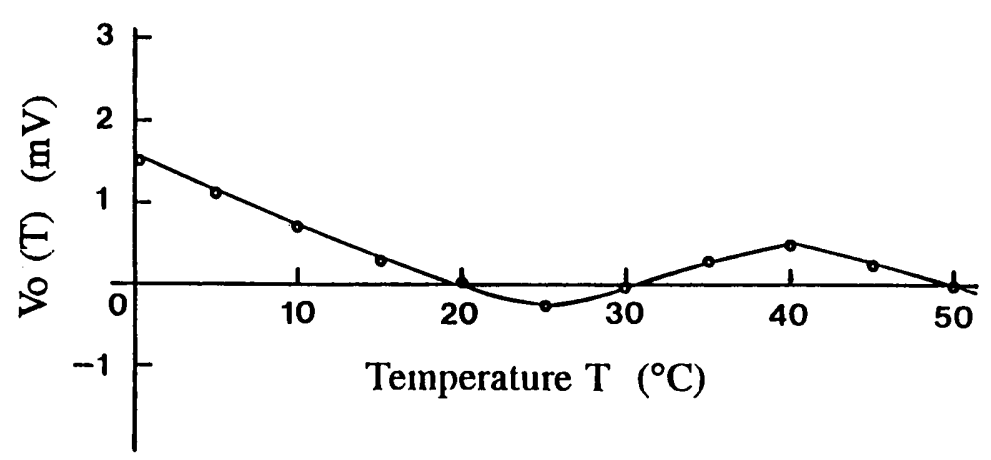

(b)

Fig. 3. (a) Signal output and (b) temperature drift of the pressure gauge using piezoresistive sensors arranged in a full bridge.

perature $T$ can be derived using (6), (8), and (11):

$$
\begin{aligned}
V_{o}(T)= & \frac{R_{6}}{R_{5}}\left\{\left(1+\frac{R_{1}}{R_{2}}\right) \alpha_{12}-\left(1+\frac{R_{3}}{R_{4}}\right) \alpha_{32}\right\} \\
& \cdot \frac{\epsilon_{12}}{\alpha_{12} \Delta T_{m}+\epsilon_{12}}\left(T_{m}-T\right) .
\end{aligned}
$$

To compare the performance of this bridge with that of a conventional one, we assume now the same amplification factor, i.e., $R_{1} / R_{2}=R_{3} / R_{4}$, for the two outputs. Then (12) is reduced to

$$
\frac{V_{o}(T)}{V_{c}(T)}=\frac{\epsilon_{12}}{\alpha_{12} \Delta T_{m}+\epsilon_{12}}
$$

where

$$
V_{c}(T)=\frac{R_{6}}{R_{5}}\left(1+\frac{R_{1}}{R_{2}}\right)\left(\alpha_{12}-\alpha_{32}\right)\left(T_{m}-T\right)
$$

corresponds to the temperature drift of a conventional bridge followed by a differential amplifier with the gain $\left(1+R_{1} / R_{2}\right) R_{6} / R_{5}$. Therefore, the right-hand side of (13) represents the rejection ratio of the temperature drift (TDRR). As the TDRR increases with the temperature coefficient of the resistive sensor, the bridge circuit described here is especially useful for those sensors which make use of semiconductor piezo- and magneto-resistance effects.

\section{Application Example}

The bridge circuit described above was applied to signal processing of a commercial piezoresistive pressure sensor. The sensor consists of four resistors diffused onto a Si diaphragm and arranged in a full-bridge configuration. The resistance of each sensor is about $5 \mathrm{k} \Omega$. Metal film resistors with a low temperature coefficient are used for $R_{A}$ and $R_{B}$. The bridge is biased by a dc voltage of $8 \mathrm{~V}$. The pressure sensitivities $\zeta_{12}$ and $\zeta_{32}$ of the bridge are $-4.5 \mathrm{mV} / \mathrm{kg}$ $\mathrm{cm}^{-2}$ and $13.125 \mathrm{mV} / \mathrm{kg} \mathrm{cm}^{-2}$, and the temperature sensitivities $\alpha_{12}$ and $\alpha_{32}$ are $-6.56 \times 10^{-2} \mathrm{mV} /{ }^{\circ} \mathrm{C}$ and $-2.5 \times 10^{-2} \mathrm{mV} /{ }^{\circ} \mathrm{C}$, respectively. The null adjustment of the bridge and the gain adjustment for the temperature balance are carried out at $T_{o}=0{ }^{\circ} \mathrm{C}$ and $T_{m}=50^{\circ} \mathrm{C}$, respectively, according to the procedure described in the previous section. The gains of $A_{1}$ and $A_{4}$ are 17 and 3 , respectively.

The output voltages against applied pressure and temperature are plotted in Figs. 3(a) and 3(b), respectively. Though the bridge was first brought into balance at $T_{o}=0^{\circ} \mathrm{C}$, the balance output voltage appears at $0^{\circ} \mathrm{C}$. This is due to the interaction between the offset cancellation and the temperature balance, as described in the previous section. Despite the imperfect cancellation, the temperature drift over a temperature span from $0^{\circ} \mathrm{C}$ to $50^{\circ} \mathrm{C}$, which would otherwise reach $106 \mathrm{mV}$ in a conventional bridge with equivalent amplification, is less than $2 \mathrm{mV}$. The TDRR is thus higher than 35 $\mathrm{dB}$. This result proves the present scheme useful for a high sensitivity sensing bridge.

\section{Conclusions}

A bridge circuit was proposed which allows the cancellation of temperature drifts of resistive sensors without degrading the sensitivity. The adjustment is easy because the cancellation condition can be established independently of the bridge null balance. The price to be paid for these merits is only the use of one extra opamp. Therefore, the simple idea described herein will find wide applicability in sensor signal processing.

\section{REFERENCES}

[1] B. M. Oliver and J. M. Cage, Electronic Measurements and Instrumentation. New York: McGraw-Hill, 1971, chap. 9.

[2] C. D. Johnson and H. Al Richeh, "Highly accurate resistance deviation to frequency converter with programmable sensitivity and resolution,"' IEEE Trans. Instrum. Meas., vol. IM-35, pp. 178-181, June 1986.

[3] A. Boros, Electrical Measurements in Engineering. Amsterdam: Elsevier, 1985, chap. 6.

[4] C. D. Johnson and C. Chen, "Bridge-to-computer data acquisition system with feedback nulling,"' IEEE Trans. Instrum. Meas., vol. 39, pp. 531-534, June 1990. 